Research Article

\title{
Combined effects of space porosity and wall properties on a compressible maxwell fluid with MHD peristalsis
}

\author{
Islam M. Eldesoky ${ }^{1,2}\left(\right.$ Mohammed S. Nayel $^{3} \cdot$ Abdallah A. Galal $^{3} \cdot$ Hagar M. Raslan ${ }^{3}$
}

Received: 8 May 2020 / Accepted: 10 November 2020 / Published online: 1 December 2020

(c) Springer Nature Switzerland AG 2020

\begin{abstract}
In this research, the peristaltic motion of viscous Maxwell fluid is analyzed during its flow in a porous medium in axisymmetric tube with complaint wall to which a fixed magnetic field is applied. A perturbation technique was used to solve the governing equations of this problem with small $\varepsilon$ "amplitude ratio." The net flow rate is calculated and discussed graphically under various physical parameters. It was found that the magnetic parameter, permeability parameter of porous medium, slip effect, wall properties, relaxation time, and the compressibility of the fluid have a significant impact on the net flow rate and the reversal flow.
\end{abstract}

Keywords Peristalsis $\cdot$ MHD $\cdot$ Wall properties $\cdot$ Porous medium $\cdot$ Maxwell model

\section{List of symbols}

$R \quad$ Tube radius

a Amplitude of travelling wave

$\lambda \quad$ Wavelength

c Wave speed

$t \quad$ Time

$r \quad$ Radial coordinate

$z \quad$ Axial coordinate

$v_{r}, v_{z} \quad$ The velocity components along $r$ and $z$ direction

$B_{o} \quad$ Uniform magnetic field

$\rho \quad$ Fluid density

$p \quad$ Fluid pressure

$\vec{v} \quad$ Velocity vector

$\vec{f} \quad$ Resistance flow in porous medium

$\vec{J} \quad$ Electric current density

$\vec{B} \quad$ Magnetic induction vector

$\tilde{\tau} \quad$ Viscous stress tensor

$K_{o} \quad$ Permeability parameter

$\varphi \quad$ Porosity of porous medium

$\mu \quad$ Dynamic viscosity

$t_{m} \quad$ Relaxation time $k^{*} \quad$ Fluid compressibility factor

$\rho_{o} \quad$ The constant density at the reference pressure $p_{o}$

$L(\eta) \quad$ An operator that represent the motion of the surface of the stretched membrane

$p_{c} \quad$ The pressure outside the wall

$T \quad$ Longitudinal tension per unit width of the wall

$m \quad$ Mass per unit area

$D \quad$ Damping coefficient

$B \quad$ Flexural rigidity of the plate

$K \quad$ Spring stiffness

$\alpha \quad$ Wave number

$\varepsilon \quad$ Amplitude ratio

Re Reynolds number

$\chi \quad$ Compressibility parameter

kn Slip parameter

M Magnetic parameter

$\triangle$ Islam M. Eldesoky, eldesokyi@yahoo.com | 'Department of Basic Engineering Sciences, Faculty of Engineering, Menofia University, Shibin El Kom, Egypt. ${ }^{2}$ Department of Basic Sciences, Menofia Higher Institute of Engineering and Technology, Menofia, Egypt. ${ }^{3}$ Department of Physics and Engineering Mathematics, Faculty of Engineering, Tanta University, Tanta, Egypt. 


\section{Introduction}

Peristaltic transport is the process of pumping biological fluids through the tubes due to the presence of transverse progressive waves on the walls. Peristalsis is the primary method for transporting physiological fluids through many biological systems. There are many applications for this property in the human body, some of which are urine flow in the ureter, chyme movement through the digestive system, and the transport of nutrients from the mouth through the esophagus. There are also man-made tools as an application of peristaltic pumping process, such as mechanical pumps for physiological fluid pumping. At the beginning of the study of peristaltic motion, hypotheses were made for fluids that were classified as Newtonian fluids as Fung and Yih [1] discussed the peristaltic pumping of Newtonian fluid through a Circular Cylindrical Tube. The Newtonian classification is limited only to the study of urine flow through the ureter or fluid flow through channels/tubes under various assumptions. But in nature the behavior of non-Newtonian fluids is dominant. Most of the biological fluids in the human body, such as chyme in the digestive system and blood flow in arteries, are classified as nonNewtonian fluids which result in unexpected changes in the fluid behavior. The mathematical equations describing this problem are complex, and for this reason challenges were made by mathematicians and physicists to search for how to find numerical and analytical solutions. Raju and Devanathan [2] investigated the first attempt to study peristaltic locomotion of a non-Newtonian power low model. Srivastava and Srivastava [3] introduced the peristaltic motion of blood as a Casson model. Rajashekhar et al. [4] introduced a Herschel-Bulkley fluid which moved peristaltically in a tube. Ali et al. [5] studied the flow of Maxwell fluid which moves peristaltically in elastic channel. Several researchers handled the peristaltic motion in different configurations and geometries. Zhang et al. [6] introduced the flow of a couple stress fluid with suspended particles peristaltically under the effect of mass and heat transfer in a channel. Bhatti et al. [7] illustrated the entropy analysis of Williamson fluid which moved peristaltically in a channel under the influences of heat transfer and magnetic field.

The current study presents the non-Newtonian fluid as a Maxwell model due to it being the simplest class of viscoelastic fluids and it represents the flow of blood through the small vessels as the dimensionless relaxation time is small and at low shear stress. Tsiklauri and Beresnev [8] analyzed the peristaltic flow of Maxwellian fluid through a tube. Elkoumy et al. [9] have discussed the peristaltic motion of Maxwell fluid through porous medium under the influence of the magnetic field and the hall effect. Abbasi et al. [10] investigated the peristaltic flow of convected Maxwell fluid by taking the compressibility of the fluid into account.

Porosity has many applications in the human body and it is of great importance for understanding the mechanisms of flow in the gallbladder and lungs and blood flow through small veins and narrow arteries. There are many muscles in the human body that have porous structures, tumors are also porous masses that are penetrated by blood vessels, and the accumulation of blood that clogs the arteries and allocation of cholesterol are natural implementations for the porous medium. The study of blood flow through small arteries is what attracted scientists recently due to the fact that the arterial walls have porous properties that leads to slide on the boundaries, which affects the non-Newtonian nature of blood. The slip effect is evident when liquids move through flexible geometric shapes, such as blood flow through blood vessels. Also, the presence of an external magnetic field has a significant effect on blood flow, which helps doctors during surgeries and magnetic resonance imaging to control blood flow. Mekheimer et al. [11] introduced the peristaltic motion of Maxwell fluid in microchannel under the effect of space porosity and constant magnetic field. Rajashekhar et al. [12] illustrated the combined effect of thermal conductivity and variable viscosity on the peristaltic motion of Casson fluid in a tube through porous medium. Vaidya et al. [13] analyzed the impact of liquid properties on a peristaltic locomotion of a Rabinowitsch liquid in a channel with convective porous wall. Zhang et al. [14] investigated the entropy analysis of blood flow under the effect of magnetic field and slip conditions through a wavy channel.

Most of the investigations confirmed that one of the most important factors that affects the fluid flow is its compressibility. Aarts and Oams [15] investigated the impact of ultrasonic radiation and porosity on a compressible liquid flow in an axisymmetric cylindrical tube, and this study has been developed by Tsiklauri and Bresenev [8] by considering that the fluid is non-Newtonian.

The walls of the tube in the peristalsis are characterized by elasticity; therefore, the effects of damping, elasticity, and stiffness of the walls are necessary to study their effect on flow. In recent research, attention has been given to the effect of complaint wall on fluid motion. Elnaby and Haroun [16] discussed the peristaltic pumping of a viscous incompressible fluid through porous medium in a duct with complaint wall. Hina [17] introduced the combined impact of magnetohydrodynamic and wall properties on the peristaltic flow of Eyring-Powell fluid. Eldesoky et al. [18] illustrated the 
influences of slip flow, relaxation time, and compressibility of the liquid on the peristaltic locomotion of Maxwell fluid flow through channel and tube with complaint walls.

It is noted that no attempts were made by researchers to discuss the MHD peristaltic motion of a viscous non-Newtonian Maxwell fluid through porous medium in a tube with complaint wall by taking the compressibility of the fluid and slip flow into account. Therefore, the purpose of this research is to fill this gap. In this research, we extended the study that has been represented from Eldesoky [19] by taking the influences of a magnetic field and the wall properties of the tube. The results obtained are in agreement with the results of Eldesoky [19] by neglecting the influence of the magnetic field and the wall properties. This work is organized as follows. Section 2 includes the dimensionless governing equations while Sect. 3 presents the solution to the problem using the perturbation technique. Section 4 contains the numerical results and discussion. Finally, Sect. 5 presents a summary of the results of the paper. The results of this work are greatly beneficial to understand and clarify the blood response during the previous conditions, which helps doctors to better know and predict the flow of blood through small arteries during surgery.

This study is a theoretical and mathematical study that approaches the real life biological application and gives indication to the behavior of the blood motion under the influence of the important physical parameters such as the magnetic field effect as MRI (magnetic resonance units) and the porosity represents the fats in the arteries which make a stenosis. The relaxation time appears in the muscles relaxation and contraction of the esophagus and also the ureter that transports the urine from the kidney to the bladder. The real dimensions are much specified and it is very difficult to take these real dimensions but the authors here depend on the dimensionless analysis for the simplification. And the results give indication to the flow behavior as the elastic artery wall damping reduces the blood velocity and the magnetic flux also causes the reversal blood flow. The fats accumulation causes the porosity which restricts the motion and raises the blood pressure which can affect the heart negatively.

\section{Formulation of the problem}

Assume a compressible and viscous Maxwell fluid flow into the tube having a radius $\boldsymbol{R}$ with complaint wall through porous medium. The flow is induced by the presence of a transverse wave on the wall of the tube shown in Fig. 1. The wave shape is in the form according to [15].

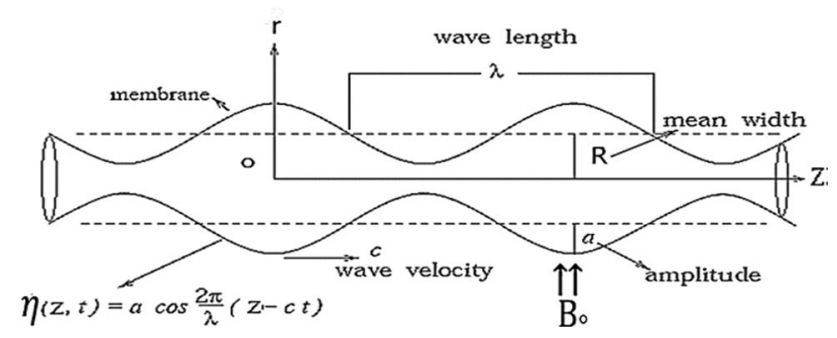

Fig. 1 Geometry of the problem

$W(z, t)=R+\eta(z, t)$,

and

$\eta(z, t)=a \cos \left(\frac{2 \pi}{\lambda}(z-c t)\right)$,

were $a$ is the amplitude of travelling wave, $\lambda$ is wavelength, and $c$ the velocity of wave.

A uniform magnetic field $B_{o}$ is directed perpendicular to the axis of the tube and assume neglecting the induced electric field. There are the polar coordinators $(r, z)$ where $z$ in the direction of axis of the tube and the velocity components are denoted $v_{r}(r, z, t)$ and $v_{z}(r, z, t)$ along $r$ direction and $z$ direction, respectively.

The governing equations of the fluid flow given by, as following [20].

\section{Conservation of mass}

$\frac{\partial \rho}{\partial t}+\nabla \cdot(\rho \vec{v})=0$,

Momentum equations

$\rho \frac{\partial \vec{v}}{\partial t}+\rho(\vec{v} \cdot \nabla) \vec{v}=-\nabla p-\nabla \cdot \tilde{\tau}+\vec{f}+\vec{J} \times \vec{B}$

where $\rho$ is the fluid density, $p$ is the fluid pressure, $\vec{v}, \vec{f}, \vec{J}, \vec{B}$ and $\tilde{\tau}$ are the velocity vector, the resistance flow in porous medium, electric current density, magnetic induction vector, and viscous stress tensor, respectively.

In Maxwell fluid, the expression of Darcy's resistance [21] as the following

$\left(1+t_{m} \frac{\partial}{\partial t}\right) \vec{f}=-\frac{\mu \varphi}{K_{o}} \vec{v}$,

where $K_{o}, \varphi$ are the permeability and porosity of the porous medium. When $\boldsymbol{B}_{\boldsymbol{o}}$ a uniform magnetic field is applied on the transverse direction of the tube, the induced electric field is ignored in case of small Reynolds number. So the magnetic field only distributes to the 
current density $\overrightarrow{\boldsymbol{J}}$ and the total magnetic body force $\overrightarrow{\boldsymbol{J}} \times \overrightarrow{\boldsymbol{B}}$ reduced to $\sigma(\overrightarrow{\boldsymbol{V}} \times \overrightarrow{\boldsymbol{B}}) \times \overrightarrow{\boldsymbol{B}}$ because there is no external electric field, where $\sigma$ is the electrical conductivity of the fluid.

$\overrightarrow{F_{e}}=\vec{J} \times \vec{B}=-\sigma B_{o}^{2} v_{z}$.

On the basis of Maxwell model [22], we assumed that

$t_{m} \frac{\partial \tilde{\tau}}{\partial t}=-\mu \nabla \vec{v}-\frac{\mu}{3}(\nabla \cdot \vec{v})-\tilde{\tau}$

where $\mu$ is the dynamic viscosity, and $t_{m}$ is the relaxation time.

Apply the operator $\left(1+t_{m} \frac{\partial}{\partial t}\right)$ to the momentum equations and eliminate $\tilde{\tau}$ using Eq. (2.5) hence, they become

$$
\begin{aligned}
(1+ & \left.t_{m} \frac{\partial}{\partial t}\right)\left(\rho \frac{\partial \vec{v}}{\partial t}+\rho(\vec{v} \cdot \nabla) \vec{v}\right) \\
= & -\left(1+t_{m} \frac{\partial}{\partial t}\right) \nabla p+\mu \nabla^{2} \vec{v}+\frac{\mu}{3} \nabla(\nabla . \vec{v}) \\
& -\frac{\mu \varphi}{K_{o}} \vec{v}+\left(1+t_{m} \frac{\partial}{\partial t}\right)(\vec{J} \times \vec{B}) .
\end{aligned}
$$

The compressibility of the fluid is described by the following equation according to Anderson [23]

$\frac{1}{\rho} \frac{\partial \rho}{\partial p}=k^{*}$,

where $k^{*}$ is the compressibility of the fluid. The solution of Eq. (2.7a) in order to the density becomes as a function of pressure is given by

$\rho=\rho_{o} e^{\left[k^{*}\left(p-p_{0}\right)\right]}$,

where $\rho_{o}$ is the constant density at the reference pressure $p_{o}$.

The governing equation of the flexible wall motion is given by [24]

$L(\eta)=p-p_{c}=-T \frac{\partial^{2} \eta}{\partial z^{2}}+m \frac{\partial^{2} \eta}{\partial t^{2}}+D \frac{\partial \eta}{\partial t}+B \frac{\partial^{4} \eta}{\partial z^{4}}+K \eta$, in which, $L$ is an operator that represents the motion of the surface of the stretched membrane with damping forces, $p_{c}$ is the pressure outside the wall coming from the tension on the muscles, $T, m, D, B, K$ are the longitudinal tension per unit width of the wall, mass per unit area, the damping coefficient of the wall, the flexural rigidity of the plate, and the spring stiffness, respectively.

According to polar coordinates system, the governing equations of the motion (2.2a), (2.2b) became

$\frac{\partial \rho}{\partial t}+v_{r} \frac{\partial \rho}{\partial r}+v_{z} \frac{\partial \rho}{\partial z}+\rho\left(\frac{\partial v_{r}}{\partial r}+\frac{v_{r}}{r}+\frac{\partial v_{z}}{\partial z}\right)=0$,

$$
\begin{aligned}
(1+ & \left.t_{m} \frac{\partial}{\partial t}\right)\left(\rho \frac{\partial v_{r}}{\partial t}+\rho\left(v_{r} \frac{\partial v_{r}}{\partial r}+v_{z} \frac{\partial v_{r}}{\partial z}\right)\right) \\
= & -\left(1+t_{m} \frac{\partial}{\partial t}\right) \frac{\partial p}{\partial r}+\mu\left(\frac{\partial^{2} v_{r}}{\partial r^{2}}+\frac{1}{r} \frac{\partial v_{r}}{\partial r}-\frac{v_{r}}{r^{2}}+\frac{\partial^{2} v_{r}}{\partial z^{2}}\right) \\
& +\frac{\mu}{3} \frac{\partial}{\partial r}\left(\frac{\partial v_{r}}{\partial r}+\frac{v_{r}}{r}+\frac{\partial v_{z}}{\partial z}\right)-\frac{\mu \varphi}{K_{o}} v_{r} \\
(1+ & \left.t_{m} \frac{\partial}{\partial t}\right)\left(\rho \frac{\partial v_{z}}{\partial t}+\rho\left(v_{r} \frac{\partial v_{z}}{\partial r}+v_{z} \frac{\partial v_{z}}{\partial z}\right)\right) \\
= & -\left(1+t_{m} \frac{\partial}{\partial t}\right) \frac{\partial p}{\partial z}+\mu\left(\frac{\partial^{2} v_{z}}{\partial r^{2}}+\frac{1}{r} \frac{\partial v_{z}}{\partial r}+\frac{\partial^{2} v_{z}}{\partial z^{2}}\right) \\
& +\frac{\mu}{3} \frac{\partial}{\partial z}\left(\frac{\partial v_{r}}{\partial r}+\frac{v_{r}}{r}+\frac{\partial v_{z}}{\partial z}\right)-\frac{\mu \varphi}{K_{o}} v_{z}-\left(1+t_{m} \frac{\partial}{\partial t}\right) \sigma B_{o}^{2} v_{z} .
\end{aligned}
$$

Taking the nature of compliant wall in account, and slip boundary condition at the boundary of the tube $(r=R+\eta)$, so the appropriate boundary conditions are

$v_{r}(W, z, t)=\frac{\partial W}{\partial t}, v_{z}(W, z, t)=A \frac{\partial v_{z}}{\partial r}$.

$\frac{\partial L(\eta)}{\partial z}=\frac{\partial p}{\partial z}$

using Eq. (2.9c) we can rewrite the pervious boundary condition (2.11a) to became

$$
\begin{aligned}
(1+ & \left.t_{m} \frac{\partial}{\partial t}\right) \frac{\partial}{\partial z}\left(-T \frac{\partial^{2} \eta}{\partial z^{2}}+m \frac{\partial^{2} \eta}{\partial t^{2}}+D \frac{\partial \eta}{\partial t}+B \frac{\partial^{4} \eta}{\partial z^{4}}+K \eta\right) \\
= & \mu\left(\frac{\partial^{2} v_{z}}{\partial r^{2}}+\frac{1}{r} \frac{\partial v_{z}}{\partial r}+\frac{\partial^{2} v_{z}}{\partial z^{2}}\right)+\frac{\mu}{3} \frac{\partial}{\partial z}\left(\frac{\partial v_{r}}{\partial r}+\frac{v_{r}}{r}+\frac{\partial v_{z}}{\partial z}\right) \\
& -\left(1+t_{m} \frac{\partial}{\partial t}\right)\left(\rho \frac{\partial v_{z}}{\partial t}+\rho\left(v_{r} \frac{\partial v_{z}}{\partial r}+v_{z} \frac{\partial v_{z}}{\partial z}\right)\right)-\frac{\mu \varphi}{K_{o}} v_{z}-\left(1+t_{m} \frac{\partial}{\partial t}\right) \sigma B_{o}^{2} v_{z} .
\end{aligned}
$$


By introducing the non-dimensional variables

where

$\bar{W}=\frac{W}{R}, \overline{v_{r}}=\frac{v_{r}}{c}, \overline{v_{z}}=\frac{v_{z}}{c}, \bar{\rho}=\frac{\rho}{\rho_{o}}, \bar{p}=\frac{p}{\rho_{o} c^{2}}, \bar{t}=\frac{t c}{R}, \bar{m}=\frac{m}{\rho_{o} R}, \bar{T}=\frac{T R \rho_{o}}{\mu^{2}}$,

$\bar{D}=\frac{D R}{\mu}, \bar{B}=\frac{B \rho_{o}}{R \mu^{2}}, \bar{K}=\frac{K R^{3} \rho_{o}}{\mu^{2}}, \overline{K_{o}}=\frac{K_{o}}{\varphi R e^{2}}$.

Non-dimensional parameters are given by

$\alpha=\frac{2 \pi R}{\lambda}, \varepsilon=\frac{a}{R}, R e=\frac{\rho_{o} c R}{\mu}, \chi=k^{*} \rho_{o} c^{2}, k n=\frac{A}{R}, M=\frac{R \sigma B_{0}^{2}}{\rho_{o} c}$,

(2.12b)

which $\alpha$ represent wave number, $\varepsilon$ is amplitude ratio, $R e$ is Reynolds number, $\chi$ is the compressibility parameter, $k n$ is the slip parameter, $M$ is he magnetic parameter.

In dimensionless form Eqs. (2.7b), (2.9) reduced to

$$
\begin{aligned}
& \frac{\partial \rho}{\partial t}+ v_{r} \frac{\partial \rho}{\partial r}+v_{z} \frac{\partial \rho}{\partial z}+\rho\left(\frac{\partial v_{r}}{\partial r}+\frac{v_{r}}{r}+\frac{\partial v_{z}}{\partial z}\right)=0 \\
&\left(1+t_{m} \frac{\partial}{\partial t}\right)\left(\rho \frac{\partial v_{r}}{\partial t}+\rho\left(v_{r} \frac{\partial v_{r}}{\partial r}+v_{z} \frac{\partial v_{r}}{\partial z}\right)\right) \\
&=-\left(1+t_{m} \frac{\partial}{\partial t}\right) \frac{\partial p}{\partial r}+\frac{1}{\operatorname{Re}}\left(\frac{\partial^{2} v_{r}}{\partial r^{2}}+\frac{1}{r} \frac{\partial v_{r}}{\partial r}-\frac{v_{r}}{r^{2}}+\frac{\partial^{2} v_{r}}{\partial z^{2}}\right) \\
&+\frac{1}{3 \operatorname{Re}} \frac{\partial}{\partial r}\left(\frac{\partial v_{r}}{\partial r}+\frac{v_{r}}{r}+\frac{\partial v_{z}}{\partial z}\right)-\frac{v_{r}}{K_{o} R^{\prime}}
\end{aligned}
$$$$
\left(1+t_{m} \frac{\partial}{\partial t}\right)\left(\rho \frac{\partial v_{z}}{\partial t}+\rho\left(v_{r} \frac{\partial v_{z}}{\partial r}+v_{z} \frac{\partial v_{z}}{\partial z}\right)\right)
$$$$
=-\left(1+t_{m} \frac{\partial}{\partial t}\right) \frac{\partial p}{\partial z}+\frac{1}{R e}\left(\frac{\partial^{2} v_{z}}{\partial r^{2}}+\frac{1}{r} \frac{\partial v_{z}}{\partial r}+\frac{\partial^{2} v_{z}}{\partial z^{2}}\right)
$$$$
+\frac{1}{3 R e} \frac{\partial}{\partial z}\left(\frac{\partial v_{r}}{\partial r}+\frac{v_{r}}{r}+\frac{\partial v_{z}}{\partial z}\right)-\frac{v_{z}}{K_{o} R e}-\left(1+t_{m} \frac{\partial}{\partial t}\right) M v_{z^{\prime}}
$$

$\rho=\rho_{o} e^{\left[k^{*}\left(p-p_{0}\right)\right]}$.

Also, boundary conditions (2.10), (2.11b) became

$$
\begin{aligned}
& v_{r}((1+\zeta), z, t)=\frac{\partial \zeta(z, t)}{\partial t}, v_{z}((1+\zeta), z, t)=k n \frac{\partial v_{z}(r, z, t)}{\partial r}, \\
& \left(1+t_{m} \frac{\partial}{\partial t}\right) \frac{\partial}{\partial z}\left(-\frac{T}{R e^{2}} \frac{\partial^{2} \zeta}{\partial z^{2}}+m \frac{\partial^{2} \zeta}{\partial t^{2}}+\frac{D}{R e} \frac{\partial \zeta}{\partial t}+\frac{B}{R e^{2}} \frac{\partial^{4} \zeta}{\partial z^{4}}+\frac{K \zeta}{R e^{2}}\right) \\
& =-\left(1+t_{m} \frac{\partial}{\partial t}\right)\left(\rho \frac{\partial v_{z}}{\partial t}+\rho\left(v_{r} \frac{\partial v_{z}}{\partial r}+v_{z} \frac{\partial v_{z}}{\partial z}\right)\right) \\
& +\frac{1}{R e}\left(\frac{\partial^{2} v_{z}}{\partial r^{2}}+\frac{1}{r} \frac{\partial v_{z}}{\partial r}+\frac{\partial^{2} v_{z}}{\partial z^{2}}\right)+\frac{1}{3 R e} \frac{\partial}{\partial z}\left(\frac{\partial v_{r}}{\partial r}+\frac{v_{r}}{r}+\frac{\partial v_{z}}{\partial z}\right) \\
& -\frac{v_{z}}{K_{o} R e}-\left(1+t_{m} \frac{\partial}{\partial t}\right) M v_{z^{\prime}}
\end{aligned}
$$

$$
\zeta=\varepsilon \cos \left(\frac{2 \pi}{\lambda}(z-c t)\right)
$$

\section{Method of solution}

Consider the case of no peristaltic wave (no flow) to illustrate the nature of solution. By choosing small ratio $\varepsilon \ll 1$ and using perturbation technique with small ratio $\varepsilon$. We seek the solution in the following form [15]

$p=p_{o}+\varepsilon p_{1}(r, z, t)+\varepsilon^{2} p_{2}(r, z, t)+\cdots$,

$v_{r}=\varepsilon u_{1}(r, z, t)+\varepsilon^{2} u_{2}(r, z, t)+\cdots$,

$v_{z}=\varepsilon v_{1}(r, z, t)+\varepsilon^{2} v_{2}(r, z, t)+\cdots$,

$\rho=1+\varepsilon \rho_{1}(r, z, t)+\varepsilon^{2} \rho_{2}(r, z, t)+\cdots$

Substituting the pervious expansions (3.1) into Eq. (2.13) and equating the coefficients of equal powers of on both sides of the equations, we obtain two systems of equations

For $\varepsilon$ :

$\frac{\partial \rho_{1}}{\partial t}+\frac{\partial u_{1}}{\partial r}+\frac{u_{1}}{r}+\frac{\partial v_{1}}{\partial z}=0$,

$$
\begin{aligned}
(1+ & \left.t_{m} \frac{\partial}{\partial t}\right) \frac{\partial u_{1}}{\partial t} \\
= & -\left(1+t_{m} \frac{\partial}{\partial t}\right) \frac{\partial p_{1}}{\partial r}+\frac{1}{R e}\left(\frac{\partial^{2} u_{1}}{\partial r^{2}}+\frac{1}{r} \frac{\partial u_{1}}{\partial r}-\frac{u_{1}}{r^{2}}+\frac{\partial^{2} u_{1}}{\partial z^{2}}\right) \\
& +\frac{1}{3 \operatorname{Re}} \frac{\partial}{\partial r}\left(\frac{\partial u_{1}}{\partial r}+\frac{u_{1}}{r}+\frac{\partial v_{1}}{\partial z}\right)-\frac{u_{1}}{K_{o} R e}
\end{aligned}
$$$$
\left(1+t_{m} \frac{\partial}{\partial t}\right) \frac{\partial v_{1}}{\partial t}
$$$$
=-\left(1+t_{m} \frac{\partial}{\partial t}\right) \frac{\partial p_{1}}{\partial z}+\frac{1}{R e}\left(\frac{\partial^{2} v_{1}}{\partial r^{2}}+\frac{1}{r} \frac{\partial v_{1}}{\partial r}+\frac{\partial^{2} v_{1}}{\partial z^{2}}\right)
$$$$
+\frac{1}{3 R e} \frac{\partial}{\partial z}\left(\frac{\partial u_{1}}{\partial r}+\frac{u_{1}}{r}+\frac{\partial v_{1}}{\partial z}\right)-\frac{v_{1}}{K_{o} R e}-\left(1+t_{m} \frac{\partial}{\partial t}\right) M v_{1},
$$

(2.14b) $\rho_{1}=\chi p_{1}$. 
For $\varepsilon^{2}$ :

$$
\begin{aligned}
& \frac{\partial \rho_{2}}{\partial t}+u_{1} \frac{\partial \rho_{1}}{\partial r}+v_{1} \frac{\partial \rho_{1}}{\partial z}+\frac{\partial u_{2}}{\partial r}+\frac{u_{2}}{r}+\frac{\partial v_{2}}{\partial z}+\rho_{1}\left(\frac{\partial u_{1}}{\partial r}+\frac{u_{1}}{r}+\frac{\partial v_{1}}{\partial z}\right)=0, \\
& \left(1+t_{m} \frac{\partial}{\partial t}\right)\left(\frac{\partial u_{2}}{\partial t}+\rho_{1} \frac{\partial u_{1}}{\partial t}+u_{1} \frac{\partial u_{1}}{\partial r}+v_{1} \frac{\partial u_{1}}{\partial z}\right) \\
& =-\left(1+t_{m} \frac{\partial}{\partial t}\right) \frac{\partial p_{2}}{\partial r}+\frac{1}{R e}\left(\frac{\partial^{2} u_{2}}{\partial r^{2}}+\frac{1}{r} \frac{\partial u_{2}}{\partial r}-\frac{u_{2}}{r^{2}}+\frac{\partial^{2} u_{2}}{\partial z^{2}}\right) \quad \text { For } \varepsilon: \\
& +\frac{1}{3 R e} \frac{\partial}{\partial r}\left(\frac{\partial u_{2}}{\partial r}+\frac{u_{2}}{r}+\frac{\partial v_{2}}{\partial z}\right)-\frac{u_{2}}{K_{o} R e} \\
& u_{1}(1, z, t)=\alpha \sin \alpha(z-t) \\
& \left(1+t_{m} \frac{\partial}{\partial t}\right) \frac{\partial}{\partial z}\left(\begin{array}{c}
-\frac{T \alpha^{3}}{R e^{2}} \sin \alpha(z-t)+m \alpha^{3} \sin \alpha(z-t)+\frac{D \alpha^{2}}{R e} \cos \alpha(z-t) \\
-\frac{B \alpha^{5}}{R e^{2}} \sin \alpha(z-t)-\frac{K \alpha}{R e^{2}} \sin \alpha(z-t)
\end{array}\right) \\
& =-\left(1+t_{m} \frac{\partial}{\partial t}\right) \frac{\partial v_{1}(1, z, t)}{\partial t}+\frac{1}{R e}\left(\frac{\partial^{2} v_{1}(1, z, t)}{\partial r^{2}}+\frac{\partial v_{1}(1, z, t)}{\partial r}+\frac{\partial^{2} v_{1}(1, z, t)}{\partial z^{2}}\right) \\
& +\frac{1}{3 R e} \frac{\partial}{\partial z}\left(\frac{\partial u_{1}(1, z, t)}{\partial r}+u_{1}(1, z, t)+\frac{\partial v_{1}(1, z, t)}{\partial z}\right) \\
& -\frac{v_{1}(1, z, t)}{K_{o} R e}-\left(1+t_{m} \frac{\partial}{\partial t}\right) M v_{1}(1, z, t)
\end{aligned}
$$$$
v_{1}(1, z, t)=k n \frac{\partial v_{1}(1, z, t)}{\partial r}
$$

For $\varepsilon^{2}$ :

$$
u_{2}(1, z, t)+\cos \alpha(z-t) \frac{\partial u_{1}(1, z, t)}{\partial r}=0
$$

$v_{2}(1, z, t)+\cos \alpha(z-t) \frac{\partial v_{1}(1, z, t)}{\partial r}=k n \frac{\partial}{\partial r}\left(v_{2}(1, z, t)+\cos \alpha(z-t) \frac{\partial v_{1}(1, z, t)}{\partial r}\right)$.

$$
\begin{aligned}
(1+ & \left.t_{m} \frac{\partial}{\partial t}\right)\left(\frac{\partial v_{2}}{\partial t}+\rho_{1} \frac{\partial v_{1}}{\partial t}+\left(u_{1} \frac{\partial v_{1}}{\partial r}+v_{1} \frac{\partial v_{1}}{\partial z}\right)\right) \\
= & -\left(1+t_{m} \frac{\partial}{\partial t}\right) \frac{\partial p_{2}}{\partial z}+\frac{1}{R e}\left(\frac{\partial^{2} v_{2}}{\partial r^{2}}+\frac{1}{r} \frac{\partial v_{2}}{\partial r}+\frac{\partial^{2} v_{2}}{\partial z^{2}}\right) \\
& +\frac{1}{3 R e} \frac{\partial}{\partial z}\left(\frac{\partial u_{2}}{\partial r}+\frac{u_{2}}{r}+\frac{\partial v_{2}}{\partial z}\right)-\frac{v_{2}}{K_{o} R e}-\left(1+t_{m} \frac{\partial}{\partial t}\right) M v_{2}
\end{aligned}
$$

$\rho_{2}=\chi p_{2}+\frac{\chi^{2}}{2} p_{1}^{2}$

Also, in order to getting two systems of boundary conditions, we expanded Eq. (2.14) using taylor expansion around
The solution can be chosen according to [15] in the following form

$$
\begin{aligned}
& u_{1}(r, z, t)=u_{1}(r) e^{i \alpha(z-t)}+\overline{u_{1}}(r) e^{-i \alpha(z-t)} \\
& v_{1}(r, z, t)=v_{1}(r) e^{i \alpha(z-t)}+\overline{v_{1}}(r) e^{-i \alpha(z-t)} \\
& p_{1}(r, z, t)=p_{1}(r) e^{i \alpha(z-t)}+\overline{p_{1}}(r) e^{-i \alpha(z-t)}
\end{aligned}
$$

$\rho_{1}(r, z, t)=\chi p_{1}(r) e^{i \alpha(z-t)}+\chi \bar{p}(r) e^{-i \alpha(z-t)}$, 
and

$u_{2}(r, z, t)=u_{20}(r)+u_{2}(r) e^{2 i \alpha(z-t)}+\overline{u_{2}}(r) e^{-2 i \alpha(z-t)}$,

$v_{2}(r, z, t)=v_{20}(r)+v_{2}(r) e^{2 i \alpha(z-t)}+\overline{v_{2}}(r) e^{-2 i \alpha(z-t)}$,

$p_{2}(r, z, t)=p_{20}(r)+p_{2}(r) e^{2 i \alpha(z-t)}+\overline{p_{2}}(r) e^{-2 i \alpha(z-t)}$,

$\rho_{2}(r, z, t)=D_{20}(r)+D_{2}(r) e^{2 i \alpha(z-t)}+\overline{D_{2}}(r) e^{-2 i \alpha(z-t)}$,

where the bar above the variables denotes the complex conjugate.

Substituting with the solution (3.2) in Eq. (3.1.1) and (3.1.3) we have got system of differential equations and boundary conditions

$u_{1}^{\prime}(r)+\frac{u_{1}(r)}{r}+i \alpha v_{1}(r)=i \alpha \chi p_{1}(r)$,

$$
\delta=\frac{1}{2 i R e^{2}}\left(-T \alpha^{3}+m \alpha^{3} R e^{2}+i \alpha^{2} D R e-B \alpha^{5}-K \alpha\right) .
$$

We rewrite the system of Eq. (3.2.1) in the form

$u_{1}^{\prime \prime}(r)+\frac{u_{1}^{\prime}(r)}{r}-\frac{u_{1}(r)}{r^{2}}-\beta_{1}^{2} u_{1}(r)-\gamma p_{1}^{\prime}(r)=0$

$v_{1}^{\prime \prime}(r)+\frac{v_{1}^{\prime}(r)}{r}-\beta^{2} v_{1}(r)-i \alpha \gamma p_{1}(r)=0$,

in which,

$$
\begin{gathered}
\beta_{1}^{2}=\alpha^{2}+\frac{1}{K_{o}}-i \alpha\left(1-i \alpha t_{m}\right) R e, \beta^{2}=\beta_{1}^{2}+M\left(1-i \alpha t_{m}\right) R e, \\
\gamma=\left(1-i \alpha t_{m}\right) R e-\frac{i \alpha \chi}{3} .
\end{gathered}
$$

Eliminating $v_{1}(r)$ between Eqs. (3.2.1a), (3.2.1c) we have got the following equation

$$
\begin{aligned}
& -i \alpha\left(1-i \alpha t_{m}\right) u_{1}(r)=-\left(1-i \alpha t_{m}\right) p_{1}^{\prime}(r)+\frac{1}{R e}\left(u_{1}^{\prime \prime}(r)+\frac{u_{1}^{\prime}(r)}{r}-\left(\frac{1}{r^{2}}+\alpha^{2}\right) u_{1}(r)\right) \\
& +\frac{1}{3 \operatorname{Re}} \frac{d}{d r}\left(u_{1}^{\prime}(r)+\frac{u_{1}(r)}{r}+i \alpha v_{1}(r)\right)-\frac{u_{1}(r)}{K_{o} \operatorname{Re}},
\end{aligned}
$$

$$
\begin{aligned}
& -i \alpha\left(1-i \alpha t_{m}\right) v_{1}(r)=-i \alpha\left(1-i \alpha t_{m}\right) p_{1}(r) \\
& +\frac{1}{R e}\left(v_{1}^{\prime \prime}(r)+\frac{v_{1}^{\prime}(r)}{r}-\alpha^{2} v_{1}(r)\right) \\
& +\frac{i \alpha}{3 R e}\left(u_{1}^{\prime}(r)+\frac{u_{1}(r)}{r}+i \alpha v_{1}(r)\right) \\
& -\frac{v_{1}(r)}{K_{o} R e}-M\left(1-i \alpha t_{m}\right) v_{1}(r) \text {. }
\end{aligned}
$$

B. Cs:

$$
\begin{aligned}
& u_{1}(1)=\frac{-i \alpha}{2} \\
& v_{1}(1)=k n v_{1}^{\prime}(1), \\
& v_{1}^{\prime \prime}(1)+v_{1}^{\prime}(1)+\frac{i \alpha}{3}\left(u_{1}^{\prime}(1)+u_{1}(1)\right)+i \alpha v_{1}(1)\left(\frac{4}{3} i \alpha+\left(1-i \alpha t_{m}\right) R e\right) \\
& \quad-\left(\frac{1}{K_{o}}+M\left(1-i \alpha t_{m}\right) R e\right) v_{1}(1)=\left(1-i \alpha t_{m}\right) R e \delta
\end{aligned}
$$

$$
\begin{aligned}
& \frac{-i \chi}{\alpha}\left[p_{1}^{\prime \prime}(r)+\frac{p_{1}^{\prime}(r)}{r}-\left(\beta^{2}+\frac{i \alpha \gamma}{\chi}\right) p_{1}(r)\right] \\
& +\frac{1}{\alpha^{2}}\left(\frac{1}{r}+\frac{d}{d r}\right)\left[u_{1}^{\prime \prime}(r)+\frac{u_{1}^{\prime}(r)}{r}-\frac{u_{1}(r)}{r^{2}}-\beta^{2} u_{1}(r)\right]=0,
\end{aligned}
$$

the previous Eq. (3.2.3d) has been differentiated with respect to $r$ and used Eq. (3.2.3a) to eliminate $p_{1}(r)$ and its derivatives, then reduced to be

$\left[\frac{d^{2}}{d r^{2}}+\frac{1}{r} \frac{d}{d r}-\frac{1}{r^{2}}-a_{1}^{2}\right]\left[\frac{d^{2}}{d r^{2}}+\frac{1}{r} \frac{d}{d r}-\frac{1}{r^{2}}-a_{2}^{2}\right] u_{1}(r)=0$,

where

$\left[\frac{d^{2}}{d r^{2}}+\frac{1}{r} \frac{d}{d r}-\frac{1}{r^{2}}-a_{1}^{2}\right]\left[\frac{d^{2}}{d r^{2}}+\frac{1}{r} \frac{d}{d r}-\frac{1}{r^{2}}-a_{2}^{2}\right] u_{1}(r)=0$,

The expressions of $v_{1}$ and $v$ can be written in the following form in terms of $t_{m}, \chi, \alpha, R e, K_{o}, M$

where

$v_{1}^{2}=\frac{\alpha^{2}\left[\left(1-i \alpha t_{m}\right) \operatorname{Re}(1-\chi)-\frac{4}{3}(i \alpha \chi)\right]-i \alpha \chi\left[\frac{1}{k_{o}}+M\left(1-i \alpha t_{m}\right) \operatorname{Re}\right]}{\left(1-i \alpha t_{m}\right) \operatorname{Re}-\frac{4}{3}(i \alpha \chi)}$, 
$v^{2}=\frac{\alpha^{2}\left[\left(1-i \alpha t_{m}\right) \operatorname{Re}(1-\chi)-\frac{4}{3}(i \alpha \chi)\right]-i \alpha \chi\left[\frac{1}{k_{o}}\right]}{\left(1-i \alpha t_{m}\right) \operatorname{Re}-\frac{4}{3}(i \alpha \chi)}$.

The solution that is satisfy Eq. (3.2.3e) is given by

$u_{1}(r)=c_{1} l_{1}\left(a_{1} r\right)+c_{2} l_{1}\left(a_{2} r\right)$,

where $l_{1}$ is the modified Bessel function of the first kind of order 1.

The general solution for $v_{1}(r)$ and $p_{1}(r)$ are

$v_{1}(r)=c_{1} b_{1} l_{o}\left(a_{1} r\right)+c_{2} b_{2} l_{o}\left(a_{2} r\right)$,

$p_{1}(r)=c_{1} \frac{\left(a_{1}^{2}-\beta_{1}^{2}\right)}{a_{1} \gamma} I_{o}\left(a_{1} r\right)+c_{2} \frac{\left(a_{2}^{2}-\beta_{1}^{2}\right)}{a_{2} \gamma} I_{o}\left(a_{2} r\right)$

Here, $I_{0}$ is the modified Bessel function of the first kind of order zero, and $c_{1}, c_{2}$ are complex numbers which satisfy boundary conditions (3.2.2)

$c_{1}=-\frac{b_{2} g_{2}\left(1-i \alpha t_{m}\right) \operatorname{Re} \delta}{R_{2} b_{1} g_{1}-R_{1} b_{2} g_{2}}, c_{2}=\frac{b_{1} g_{1}\left(1-i \alpha t_{m}\right) \operatorname{Re} \delta}{R_{2} b_{1} g_{1}-R_{1} b_{2} g_{2}}$,

in which,

$b_{1}=\frac{\chi\left(a_{1}^{2}-\beta_{1}^{2}\right)}{a_{1} \gamma}+\frac{i}{\alpha} a_{1}, b_{2}=\frac{\chi\left(a_{2}^{2}-\beta_{1}^{2}\right)}{a_{2} \gamma}+\frac{i}{\alpha} a_{2}$

$R_{1}=\left(\frac{i \alpha}{3} a_{1}-\left(\frac{1}{3} \alpha^{2}+\beta^{2}\right) b_{1}+a_{1}^{2} b_{1}\right) l_{o}\left(a_{1}\right), R_{2}=\left(\frac{i \alpha}{3} a_{2}-\left(\frac{1}{3} \alpha^{2}+\beta^{2}\right) b_{2}+a_{2}^{2} b_{2}\right) l_{o}\left(a_{2}\right)$,

$g_{1}=I_{o}\left(a_{1}\right)-k n a_{1} I_{1}\left(a_{1}\right), g_{2}=I_{o}\left(a_{2}\right)-k n a_{2} I_{1}\left(a_{2}\right)$.

In order to solve the second order system, inserting the Eq. (3.3) onto the partial differential Eq. (3.1.2) and their boundary conditions (3.1.4) we introduced the following equations

$D_{1}=\frac{i \alpha k n}{2}\left[c_{1} b_{1} a_{1} l_{1}\left(a_{1}\right)+c_{2} b_{2} a_{2} l_{1}\left(a_{2}\right)-\overline{c_{1}} \overline{b_{1}} \overline{a_{1}} l_{1}\left(\overline{a_{1}}\right)-\overline{c_{2}} \overline{b_{2}} \overline{a_{2}} l_{1}\left(\overline{a_{2}}\right)\right]$.

$$
\begin{aligned}
& -p_{20}^{\prime}(r)+\frac{4}{3 \operatorname{Re}}\left(u_{20}^{\prime \prime}(r)+\frac{u_{20}^{\prime}(r)}{r}-\frac{u_{20}(r)}{r^{2}}\right)-\frac{u_{20}(r)}{K_{o} R e} \\
& =i \alpha \chi\left[p_{1}(r) \overline{u_{1}}(r)-\overline{p_{1}}(r) u_{1}(r)\right]+\left[u_{1}(r) \overline{u_{1}^{\prime}}(r)+\overline{u_{1}}(r) u_{1}^{\prime}(r)\right] \\
& \quad+i \alpha\left[u_{1}(r) \overline{v_{1}}(r)-\overline{u_{1}}(r) v_{1}(r)\right],
\end{aligned}
$$

$v_{20}^{\prime \prime}(r)+\frac{v_{20}^{\prime}(r)}{r}-n^{2} v_{20}(r)=\operatorname{Re} \frac{1}{r} \frac{d}{d r}\left(r\left(u_{1}(r) \overline{v_{1}}(r)+\overline{u_{1}}(r) v_{1}(r)\right)\right)$,

$D_{20}(r)=\chi p_{20}(r)+\chi^{2} p_{1}(r) \overline{p_{1}}(r)$,

where

$n^{2}=\frac{1}{K_{o}}+$ MRe.

$u_{20}(1)+\frac{1}{2}\left(\overline{u_{1}^{\prime}}(1)+u_{1}^{\prime}(1)\right)=0$,

$v_{20}(1)+\frac{1}{2}\left(\overline{v_{1}^{\prime}}(1)+v_{1}^{\prime}(1)\right)=k n\left[v_{20}^{\prime}(1)+\frac{1}{2}\left(v_{1}^{\prime \prime}(1)+\overline{v_{1}^{\prime \prime}}(1)\right)\right]$

It will be noted that the functions $\boldsymbol{u}_{20}(\boldsymbol{r}), \boldsymbol{v}_{20}(\boldsymbol{r}), \boldsymbol{p}_{20}(\boldsymbol{r})$, and $\boldsymbol{D}_{20}(\boldsymbol{r})$ only participate in the solution of the net flow rate, as long as terms up to $O\left(\varepsilon^{2}\right)$ are retained. So $\boldsymbol{u}_{2}(\boldsymbol{r})$, where $D_{1}$ is complex number obtained according to the boundary condition (3.3.2a), which defined as

$\boldsymbol{V}_{2}(\boldsymbol{r})$, and $\boldsymbol{D}_{2}(\boldsymbol{r})$ do not participate in the net flow.

$u_{20}(r)=\frac{D_{1}}{r}-\chi\left[p_{1}(r) \overline{u_{1}}(r)+\overline{p_{1}}(r) u_{1}(r)\right]$, boundary condition (3.3.2a), which defined as $u_{20}^{\prime}(r)+\frac{u_{20}(r)}{r}+\chi\left(\frac{1}{r}+\frac{d}{d r}\right)\left[p_{1}(r) \overline{u_{1}}(r)+\overline{p_{1}}(r) u_{1}(r)\right]=0$,
Also, the solution for equation $v_{20}(r)$ have been defined as follows

$v_{20}(r)=I_{o}(n r)\left[\frac{D}{I_{o}(n)}-\operatorname{Re} \int_{r}^{1} \delta_{1}(\xi) d \xi\right]$,

where 


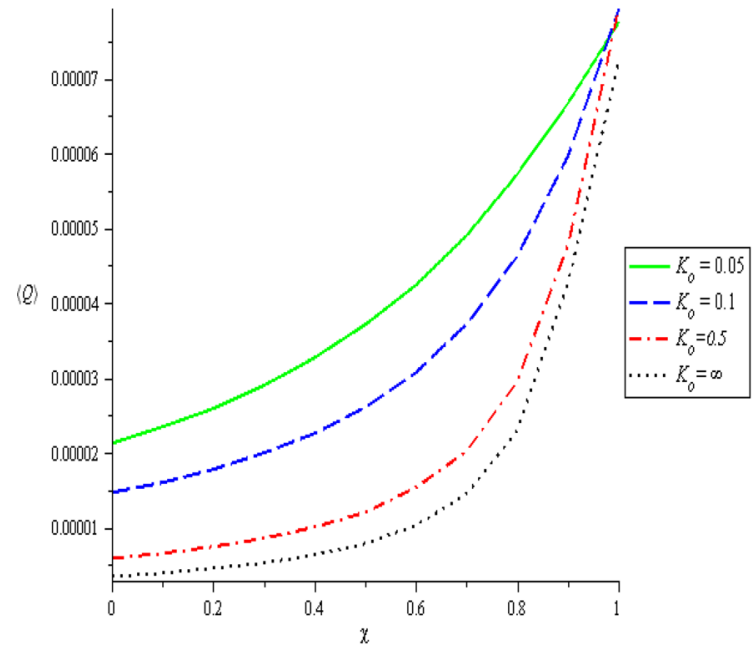

(a)

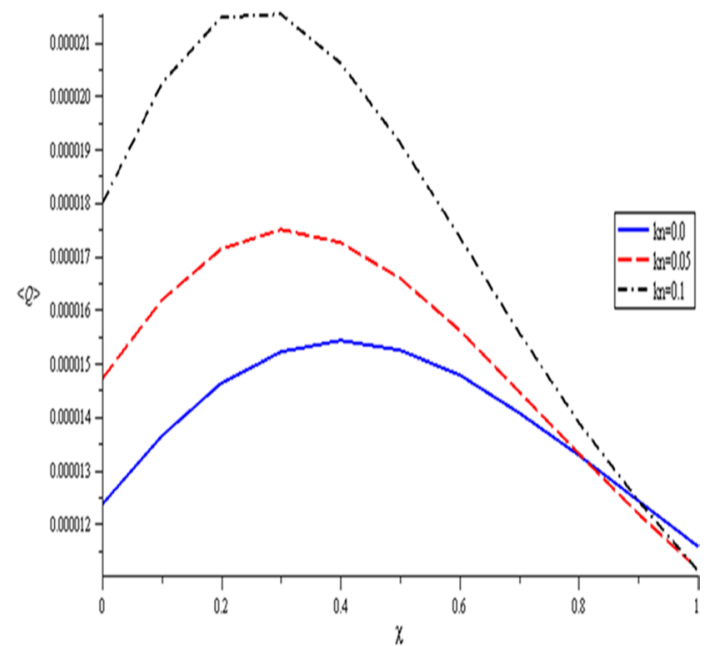

(b)

Fig. 2 The net flow rate versus compressibility parameter

$$
\begin{aligned}
& \delta_{1}(\xi)=K_{o}(n \xi)\left[\psi(\xi)+\xi \psi^{\prime}(\xi)\right], \\
& \psi(\xi)=\left[u_{1}(\xi) \overline{v_{1}}(\xi)+\overline{u_{1}}(\xi) v_{1}(\xi)\right],
\end{aligned}
$$

here $K_{o}$ is the zero-order modified Bessel functions of the second kind. $D$ is a complex constant is obtained from the boundary condition (3.3.2b), which defined by

\section{Numerical results and discussion}

The present work demonstrates the flow of blood as Maxwell model through flexible tubes under the influence of a magnetic field through a porous medium. The research is considered an extension of the study presented by Eldesoky

$D=\left[\frac{k n}{2}\left(v_{1}^{\prime \prime}(1)+\overline{v_{1}^{\prime \prime}}(1)\right)-\frac{1}{2}\left(\overline{v_{1}^{\prime}}(1)+v_{1}^{\prime}(1)\right)+\operatorname{Rekn}_{o}(n) \delta_{1}(1)\right]\left[\frac{I_{o}(n)}{I_{o}(n)-n k n l_{1}(n)}\right]$.

Next, the net flow is averaged over one period of time. We present the average of a variable $G$ over one period $T$ of time $t$ as

$\langle G\rangle=\frac{1}{T} \int_{0}^{T} G(r, z, t) d t$

Taking $T=\frac{2 \pi}{\alpha}$, then the mean net axial velocity read as

$\left\langle v_{z}\right\rangle=\varepsilon^{2} v_{20}(r)$.

By ignoring the terms of $o\left(\varepsilon^{3}\right)$, the net flow rate is given by

$\langle Q\rangle=2 \pi \varepsilon^{2}\left[\frac{D I_{1}(n)}{n I_{o}(n)}-\frac{R e}{n} \int_{0}^{1} r l_{1}(n r) \delta_{1}(r) d r\right]$.
[19] by taking into account the effects of the flexible wall properties and the presence of an external magnetic field. To verify the validity of the present research, a comparison is made with Eldesoky and Mousa [25] and Eldesoky [19]. The same results were obtained from Eldesoky and Mousa [25] by eliminating the effect of magnetic field, wall properties, and slip condition as shown in Fig. 2a which is similar to Fig. 5 in Eldesoky and Mousa [25]. Also the results of Eldesoky [19] have been obtained by neglecting the effect of magnetic field and complaint wall. We noted that Fig. $2 b$ is identical to Fig. 3 introduced by Eldesoky [19]. The data are taken according to Table 1.

The main objective of this study is to discuss the fluid behavior while it is flowing through a tube under the influence of a group of factors such as the magnetic parameter, permeability parameter of porous medium, slip effect, wall parameters, relaxation time, and the compressibility of the 
Fig. 3 Variation of the net flux and wave number $\boldsymbol{\alpha}$

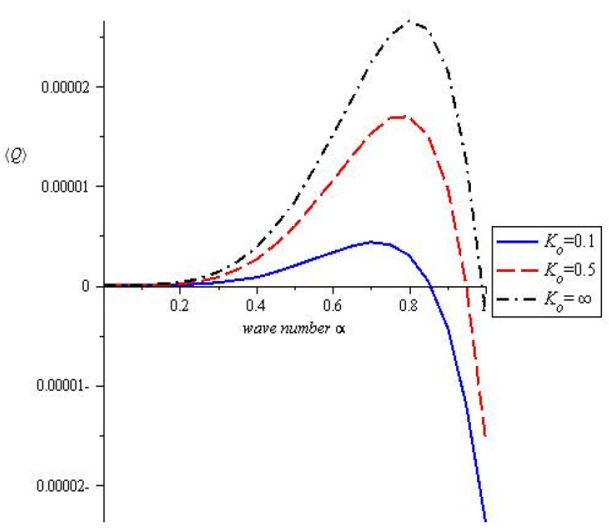

(a)

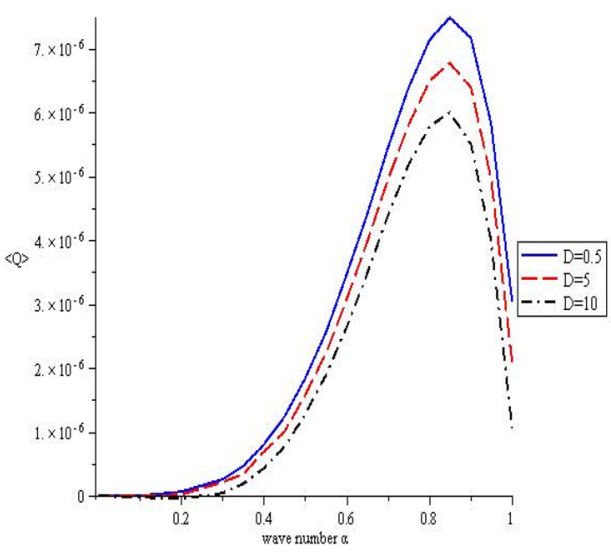

(c)

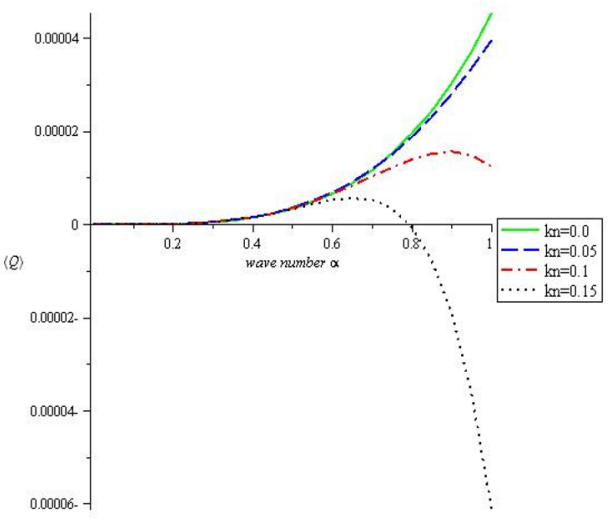

(e)

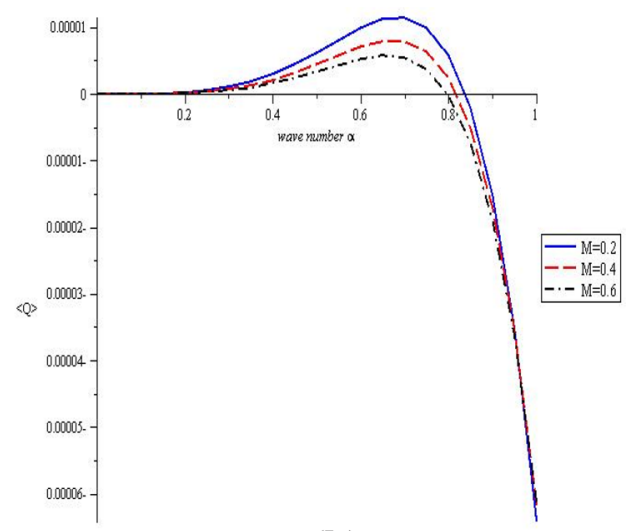

(b)

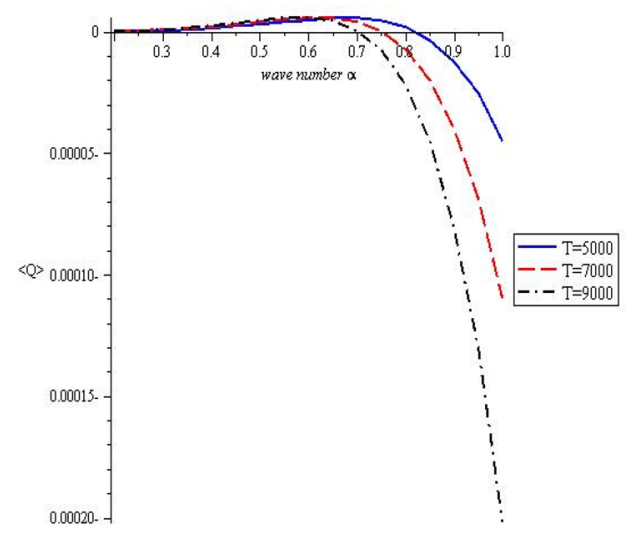

(d)

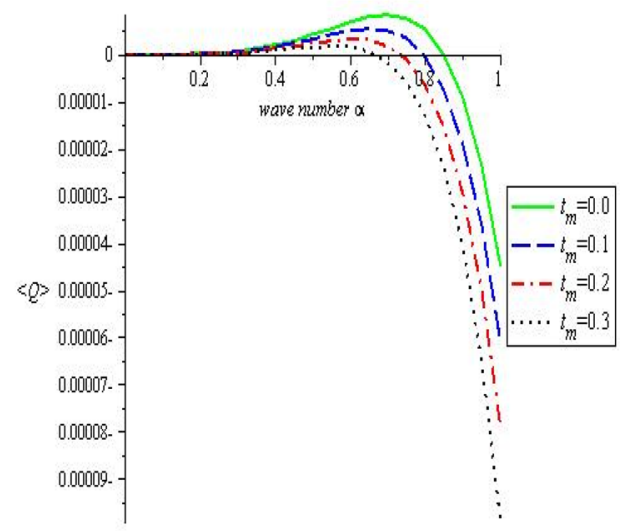

(f) fluid. Also, we introduced the expression of the mean axial velocity and the net flow rate. The net flow rate is calculated and discussed graphically under various parameters. The solution of this problem mush achieves $\varepsilon \boldsymbol{\alpha} 2 \boldsymbol{R} \boldsymbol{e} \ll 1$ according to [26]. Therefore, the fluid is considered to be laminar and the amplitude ratio is small $\varepsilon \ll 1$.
Now, we start to illustrate in Fig. 3 the relation between the net flow rate and the wave number $\alpha$ for various values of permeability parameter of porous medium " $\boldsymbol{K}_{\boldsymbol{o}}$ ", the magnetic parameter " $\boldsymbol{M}$ ", wall parameters " $\boldsymbol{D}, \boldsymbol{T}$ ", slip parameter " $\boldsymbol{k n}$ ", and relaxation time " $\boldsymbol{t}_{\boldsymbol{m}}$ ". All of them correspond to data in Table 2. 
Table 1 Corresponding to Fig. 2

\begin{tabular}{lll}
\hline & $(\mathrm{a})$ & $(\mathrm{b})$ \\
\hline$K_{o}$ & $0.05,0.1,0.5, \infty$ & 0.1 \\
$t_{m}$ & 10,000 & 1000 \\
$R e$ & 10,000 & 10,000 \\
$\varepsilon$ & 0.001 & 0.001 \\
$k n$ & 0.0 & $0.0,0.05,0.1$ \\
$\alpha$ & 0.001 & 0.001 \\
\hline
\end{tabular}

Figure 3a has shown the influence of porous medium parameter $\boldsymbol{K}_{\boldsymbol{o}}$ on the net flow rate. It is noted that increasing the porous medium parameter causes increasing the net flux and reducing the reversal flow. The relation between $\alpha, Q$ is proportional until reaching the maximum value of $\boldsymbol{Q}$ at $\boldsymbol{\alpha}=0.75$; then the relation becomes inverse and the reversal flow starts to show the effect of magnetic field parameter $\boldsymbol{M}$ represented in Fig. $3 \mathrm{~b}$. It is clear to observe that increasing $\boldsymbol{M}$ leads to reducing $\boldsymbol{Q}$ but $\boldsymbol{Q}$ grows by increasing. For all cases, $\mathbf{Q}_{\max }$ occurs at the same value of $\boldsymbol{\alpha}=0.7$, after this value $\boldsymbol{Q}$ reduces and the backward flow starts to appear. For $\alpha>0.9$ the trend of all curves converges to each other.

It is noted that in Fig. $3 c$ damping force $\boldsymbol{D}$ has a great influence on the net flow rate. The net flux rises with $\alpha$ up to $\boldsymbol{\alpha}=0.85$ and then $\boldsymbol{Q}$ falls off with $\alpha$. At certain value of $\alpha$, damping force reduces the net flow rate. The impact of wall tension $\boldsymbol{T}$ is shown in Fig. $3 \mathrm{~d}$. It is noted that, at $\alpha<0.65$, the effect of wall tension is weak; then the effect of $\boldsymbol{T}$ causes reduction in the net flux and rising in reflex flow .

The effectiveness of wall slip condition $\boldsymbol{k n}$ is clear in Fig. 3e. When $\boldsymbol{k n}=0.0,0.05$, the relation between $\boldsymbol{Q}, \boldsymbol{\alpha}$ is proportional, since at $\boldsymbol{k n}=0.1,0.15$ the relation is also proportional reaching $\boldsymbol{Q}_{\max }$ and after that the relation becomes reversal. Only at $\boldsymbol{k n}=0.15$ (full slip flow) the reversal flow exists at $\alpha=0.8$. The net flux profile versus $\boldsymbol{t}_{\boldsymbol{m}}$ is similar to the effect of damping force on $\boldsymbol{Q}$ and each of them resists the flow but the main difference of reflex flow exists for any value for relaxation time and this reflex flow rises by increasing $\boldsymbol{t}_{\boldsymbol{m}}$ as shown in Fig. 3f. Also, the value of $\boldsymbol{Q}$ does not change by varying $\boldsymbol{t}_{\boldsymbol{m}}$ as long as $\boldsymbol{\alpha} \leq 0.4$.

Figure 4 shows the distribution of the net flow rate versus the liquid compressibility for different values of relaxation time, magnetic field parameter, porous medium parameter, and wall damping coefficient. Data used in Fig. 4 are taken from Table 3.

The relaxation time $t_{m}$ considers a resistant parameter for the flow; therefore, the backward flow grows by increasing $t_{m}$ as shown in Fig. 4a. There is a raise in the flow rate by increasing $\chi$ until the maximum value of the net flux at $\chi=0.1$; then the net flow falls off and the back flow starts to exist. On the other hand, Fig. $4 \mathrm{~b}$ shows the influence of magnetic field in net flux profiles. For $\chi<0.3$, $Q$ does not change by varying the magnetic parameter; then the change in $Q$ starts to be visible; thus, it made a reduction in the net flux. $Q_{\max }=0.00001018652703$ at $\chi=0.1$ for all curves, after this value $Q$ reduces as $\chi$ increase in contrast before $\chi=0.1$.

The increased porous medium parameter strengthens the net flow as shown in Fig. 4c. At $\mathbf{K}_{\mathbf{0}}=0.1,0.15$ the reversal flow is visible and the relation between $\mathbf{Q}, \chi$ is reversal after $\chi=0.1$, but at $\mathbf{K}_{\mathbf{0}}=0.2$ the flow is positive. Finally, in Fig. $4 \mathrm{~d}$ also the trend of the flow rate under the effect of wall damping is similar to the trend of Fig. 4 a which causes a reduction in $\mathbf{Q}$.

The ranges taken in this paper for the governing parameters were selected according to the physical applications compatible with this study. The flow was classified according to Knudsen number into a continuum, transition, slip, and free molecule, whereas the
Table 2 Corresponding to Fig. 3

\begin{tabular}{lllllll}
\hline & $(\mathrm{a})$ & $(\mathrm{b})$ & $(\mathrm{c})$ & $(\mathrm{d})$ & $(\mathrm{e})$ & $(\mathrm{f})$ \\
\hline$M$ & 0.5 & $0.2,0.4,0.6$ & 1 & 0.5 & 0.5 & 0.5 \\
$K_{o}$ & $0.1,0.5, \infty$ & 1 & 0.5 & 0.5 & 0.5 & 0.5 \\
$T$ & 9866.7 & 5581 & 5581 & $5000,7000,9000$ & 5581 & 5581 \\
$D$ & 0.5 & 0.5 & $0.5,5,10$ & 0.5 & 0.5 & 0.5 \\
$K$ & 1 & 1 & 1 & 1 & 1 & 1 \\
$B$ & 2 & 2 & 2 & 2 & 2 & 2 \\
$m$ & 0.01 & 0.01 & 0.01 & 0.01 & 0.01 & 0.01 \\
$t_{m}$ & 0.1 & 0.1 & 0.1 & 0.1 & 0.1 & $0.0,0.1,0.2,0.3$ \\
$R e$ & 10 & 10 & 10 & 10 & 10 & 10 \\
$\varepsilon$ & 0.001 & 0.001 & 0.001 & 0.001 & 0.001 & 0.001 \\
$k n$ & 0.1 & 0.15 & 0.1 & 0.15 & $0.0,0.05$, & 0.15 \\
$\chi$ & 0.001 & 0.001 & 0.001 & 0.001 & $0.1,0.15$ & \\
& & & & 0.001 & 0.001 \\
\hline
\end{tabular}




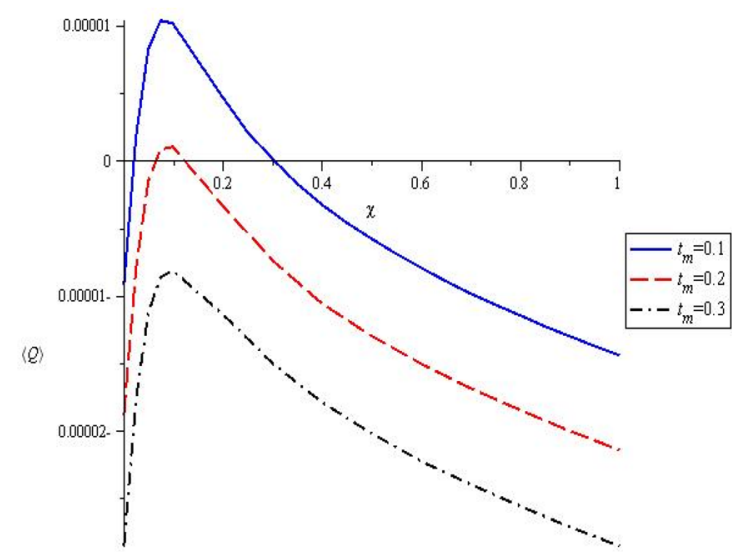

(a)

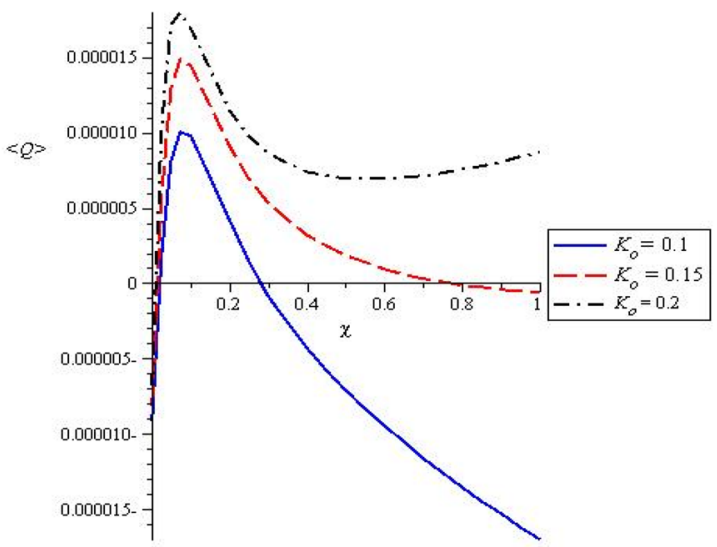

(c)

Fig. 4 Distribution of net flux versus liquid compressibility $\chi$

Table 3 Corresponding to Fig. 4

\begin{tabular}{lllll}
\hline & (a) & (b) & (c) & (d) \\
\hline$M$ & 1 & $1,3,6$ & 0.5 & 0.5 \\
$K_{0}$ & 0.2 & 0.2 & $0.1,0.15,0.2$ & 0.2 \\
$T$ & 200 & 200 & 200 & 200 \\
$D$ & 0.5 & 0.5 & 0.5 & $0.5,40,100$ \\
$K$ & 1 & 1 & 0.1 & 0.1 \\
$B$ & 20 & 20 & 20 & 20 \\
$m$ & 0.01 & 0.01 & 0.01 & 0.01 \\
$t m$ & $0.1,0.2,0.3$ & 0.1 & 0.1 & 0.1 \\
$R e$ & 0.1 & 0.1 & 0.1 & 0.1 \\
$k n$ & 0.1 & 0.1 & 0.1 & 0.1 \\
$\alpha$ & 0.6 & 0.6 & 0.6 & 0.6 \\
\hline
\end{tabular}

fluid is a continuous medium $(k n<0.01)$ if the mean free path of the molecules is neglected in comparison to the geometrical dimensions. If the fluid slips from the walls

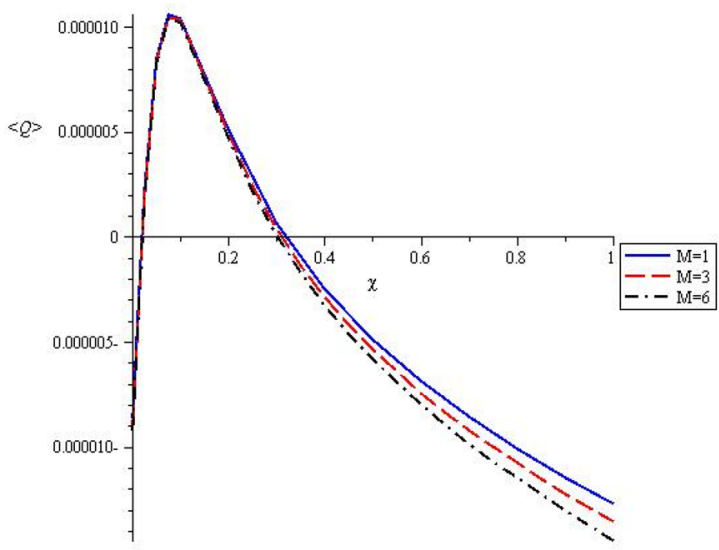

(b)

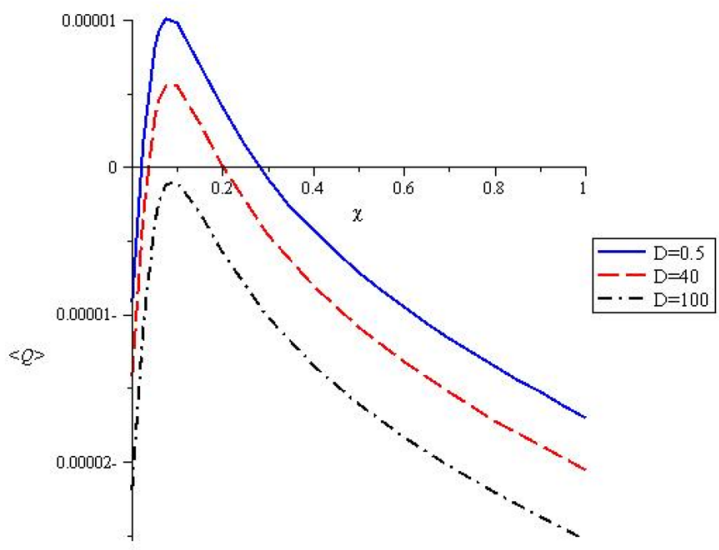

(d)

of the domain, then the range of Knudsen number is $0.01<k n<0.1$, if it is a field. When $0.1<k n<3$, then the flow exists in transition regime. The flow range exists in free molecular regime, when $k n>3$. The compressibility parameter has range $0<\chi<1$. When $\chi=0$, then the fluid is incompressible; while $\chi=1$, then flow is fully compressible. The ranges of elastic wall parameters have been chosen according to $[27,28]$.

\section{Conclusion}

This paper deals with the peristaltic flow of blood through the human circulation. The blood was represented by Maxwell fluid during its flow in a flexible tube through a porous medium and exposed to an external magnetic field. The research is looking for one of the natural phenomena, namely, the flow of blood through small arteries under 
the influence of a magnetic field. There is a possibility for developing the presented model by studying the effect of catheters on the dynamics of blood flow through the human body (catheterized artery) to help doctors control blood flow during catheter and endoscopic operations.

This study is available for the peristaltic transport with slip regime $\mathrm{kn}[0.01: 0.15]$ and compressibility factor $\chi \leq 1$, noting that at $\chi=0$ means fully incompressible flow. It is recommended to study all of these effects in the case of incompressible flow and compare it with the results of this study when $\chi=0$.

The current paper can be described as an extension of Eldesoky [19]. The peristaltic locomotion of non-Newtonian Maxwell fluid via porous tube were discussed. Moreover, a discussion of the fluid-flow behavior is presented under the influence of many parameters, for example, compressibility of the fluid, relaxation time, wave number, and the elastic wall features in the presence of porous medium and magnetic field. Section 4 shows the net flux profiles versus liquid compressibility and wave number under the impact of the aforementioned parameters and, through cancelling the impact of magnetic field and wall properties, we return to Eldesoky [19].

Our main results are outlined below:

The wall damping has an effect of reducing the net flow rate. Moreover, the wall tension has the same the same effect because of the emergence of the reversal flow. Each of the porous medium parameter and the magnetic parameter exerts a substantial effect on the net flow rate: when the porous parameter is increased, this causes the net flux to rise. On the contrary, the net flux is reduced by the magnetic parameter. Moreover, the flow rate profiles decrease; hence, the back flow is increased through increasing the relaxation time and the slip condition. The liquid's compressibility has a strong impact upon the net flux that leads the net flow rate to decrease at a greater value. There is a proportional relation between the wave number and the net flow rate, which can reach the maximum flow. Afterwards, the relation turns into an inverse relation, and the backward flow may now emerge. The upcoming research topics will handle the combined effects of nanoparticles concentrations in the cancer treatment area of research in addition to introducing the pulsation effects. Also some medical applications acquire rotating catheter tubes inside the esophagus. The effects of rotating with constant and variable angular velocity on the micropolar fluid model in a circular tube will be studied also.

\section{Compliance with ethical standards}

Conflict of interest The authors declare that they have no conflict of interest.

\section{References}

1. Fung Y, Yih C (1968) Peristaltic transport. ASME J Appl Mech 33:669-675

2. Raju K, Devanathan R (1972) Peristaltic motion of a non-Newtonian fluid. Rheol Acta 11(2):170-178

3. Srivastava L, Srivastava V (1984) Peristaltic transport of blood: Casson model-II. J Biomech 17(11):821-829

4. Rajashekhar C, Manjunatha G, Prasad K, Divya B, Vaidya H (2018) Peristaltic transport of two-layered blood flow using HerschelBulkley model. Cogent Eng 5(1):1495592

5. Ali N, Hayat T, Asghar S (2009) Peristaltic flow of a Maxwell fluid in a channel with compliant walls. Chaos Solitons Fractals 39(1):407-416

6. Zhang L, Bhatti M, Michaelides EE (2020) Thermally developed coupled stress particle-fluid motion with mass transfer and peristalsis. JTherm Anal Calorim. https://doi.org/10.1007/s1097 3-020-09871-w

7. Bhatti M, Riaz A, Zhang L, Sait SM, Ellahi R (2020) Biologically inspired thermal transport on the rheology of Williamson hydromagnetic nanofluid flow with convection: an entropy analysis. J Therm Anal Calorim. https://doi.org/10.1007/s10973-020-09876 $-5$

8. Tsiklauri D, Beresnev I (2001) Non-Newtonian effects in the peristaltic flow of a Maxwell fluid. Phys Rev E 64(3):036303

9. El Koumy SR, El Sayed IB, Abdelsalam SI (2012) Hall and porous boundaries effects on peristaltic transport through porous medium of a Maxwell model. Transp Porous Med 94(3):643-658

10. Abbasi A, Ahmad I, Ali N, Hayat T (2016) An analysis of peristaltic motion of compressible convected Maxwell fluid. AIP Adv 6(1):015119

11. Mekheimer KS, Komy SR, Abdelsalam SI (2013) Simultaneous effects of magnetic field and space porosity on compressible Maxwell fluid transport induced by a surface acoustic wave in a microchannel. Chin Phys B 22(12):124702

12. Rajashekhar C, Manjunatha G, Vaidya H, Divya B, Prasad K (2018) Peristaltic flow of Casson liquid in an inclined porous tube with convective boundary conditions and variable liquid properties. Front Heat Mass Transf (FHMT) 11:35

13. Vaidya H, Rajashekhar C, Manjunatha G, Prasad K (2019) Effect of variable liquid properties on peristaltic flow of a Rabinowitsch fluid in an inclined convective porous channel. Eur Phys J Plus 134(5):231

14. Zhang L, Bhatti MM, Michaelides EE (2020) Entropy generation in magnetized blood flow through a finite wavy channel under slip conditions. J Non-Equilib Thermody 1, no. ahead-of-print

15. Aarts A, Ooms G (1998) Net flow of compressible viscous liquids induced by travelling waves in porous media. J Eng Math 34(4):435-450

16. Elnaby MAA, Haroun MH (2008) A new model for study the effect of wall properties on peristaltic transport of a viscous fluid. Commun Nonlinear Sci Numer Simul 13(4):752-762

17. Hina $S$ (2016) MHD peristaltic transport of Eyring-Powell fluid with heat/mass transfer, wall properties and slip conditions. J Magn Magn Mater 404:148-158

18. Eldesoky IM, Abumandour RM, Abdelwahab ET (2019) Analysis for various effects of relaxation time and wall properties on 
compressible Maxwellian peristaltic slip flow. Zeitschrift für Naturforschung A 74(4):317-331

19. Eldesoky I (2012) Influence of slip condition on peristaltic transport of a compressible Maxwell fluid through porous medium in a tube. Int J Appl Math Mech 8(2):99-117

20. Salih A (2011) Conservation equations of fluid dynamics. Department of Aerospace Engineering Indian Institute of Space Science and Technology, Thiruvananthapuram

21. Hayat T, Ali N, Asghar S (2007) Hall effects on peristaltic flow of a Maxwell fluid in a porous medium. Phys Lett A 363(5-6):397-403

22. Del Rio J, De Haro ML, Whitaker S (1998) Enhancement in the dynamic response of a viscoelastic fluid flowing in a tube. Phys Rev E 58(5):6323

23. Anderson JD (1990) Modern compressible flow: with historical perspective. McGraw-Hill, New York

24. Mekheimer KS, Abdel-Wahab A (2011) Effect of wall compliance on compressible fluid transport induced by a surface acoustic wave in a microchannel. Numer Methods Part Differ Equ 27(3):621-636

25. Eldesoky IM, Mousa A (2010) Peristaltic flow of a compressible non-Newtonian Maxwellian fluid through porous medium in a tube. Int J Biomath 3(02):255-275

26. Takabatake S, Ayukawa K, Mori A (1988) Peristaltic pumping in circular cylindrical tubes: a numerical study of fluid transport and its efficiency. J Fluid Mech 193:267-283

27. Shankar V, Kumaran V (1999) Stability of non-parabolic flow in a flexible tube. J Fluid Mech 395:211-236

28. Shankar V, Kumaran V (2002) Stability of wall modes in fluid flow past a flexible surface. Phys Fluids 14(7):2324-2338

Publisher's Note Springer Nature remains neutral with regard to jurisdictional claims in published maps and institutional affiliations. 